\title{
NUMERICAL APPROXIMATION OF THE SMOLUCHOWSKI EQUATION USING RADIAL BASIS FUNCTIONS*
}

\author{
Christiane Helzel \\ Institut of Mathematics, Heinrich-Heine-Universität Düsseldorf, Germany \\ Email: christiane.helzel@hhu.de \\ Maximilian Schneiders \\ formerly Institut of Mathematics, Heinrich-Heine-Universität Düsseldorf, Germany \\ Email: Maximilian.Schneiders@hhu.de
}

\begin{abstract}
The goal of this paper is to present a numerical method for the Smoluchowski equation, a drift-diffusion equation on the sphere, arising in the modelling of particle dynamics. The numerical method uses radial basis functions (RBF). This is a relatively new approach, which has recently mainly been used for geophysical applications. For a simplified model problem we compare the RBF approach with a spectral method, i.e. the standard approach used in related physical applications. This comparison as well as our other accuracy studies show that RBF methods are an attractive alternative for these kind of models.
\end{abstract}

Mathematics subject classification: 65M20, 65M70.

Key words: Smoluchowski equation, Spectral method, Radial basis function method.

\section{Introduction}

In this paper we study a numerical method which can be used in order to simulate the microstructure in a micro-macro model for suspensions of rod-like particles. Such mathematical models are used to describe complex fluids such as polymeric fluids. The full model is a time dependent, five dimensional system of partial differential equations. It consists of a three dimensional fluid flow equation which is coupled with a transport diffusion equation on the sphere describing the microscopic orientation of the suspended rod-like particles. Here we restrict our considerations to the description of the microscopic orientation, i.e. the partial differential equation on the sphere and provide a numerical method for this equation.

Numerical methods for such complex fluids are typically based on a fully macroscopic approach or on a stochastic approach using Monte-Carlo methods. We are instead interested in numerical methods which resolve the full multiscale problem. In Helzel and Otto [8], a finite difference method for the macroscopic flow equations was combined with a finite volume method for the microscopic equation. For a related model, Knezevic and Süli [10] combined finite element and spectral methods. In this paper we show that the use of radial basis function methods provides an attractive alternative for the resolution of the microscopic transport diffusion equation.

Radial basis functions provide a powerful tool for the grid-free approximation of multivariate functions, see for example the review by Buhmann [2]. Flyer and Wright [4,5] introduced radial basis function methods for the approximation of transport dominated partial differential

\footnotetext{
* Received September 30, 2018 / Revised version received April 24, 2019 / Accepted August 30, 2019 /

Published online November 29, 2019 /
} 
equations on the sphere. Since then such methods have successfully been used for several models, mainly motivated by geophysical applications as documented in the recent book by Fornberg and Flyer [6]. The method has also been extended to solve reaction-diffusion equations on surfaces (other than the sphere), see Piret [13] and Shankar et al. [14].

In what follows, we start in Section 2 with a description of the mathematical model. In Section 3 we restrict our considerations to a simplified one-dimensional model. For this model we derive a spectral method as well as a radial basis function method and compare their performance. In Section 4 we derive the radial basis function method for the full model and discuss numerical results for this method.

\section{The Mathematical Model}

We consider a mathematical model, which describes dilute suspensions of rod-like particles. With this model we can study the dynamics of a macroscopic flow which is influenced by suspended microscopic particles with a rigid rod-like structure.

Following the classical work of Doi and Edwards [3] and more recent work of Otto and Tzavaras [12], we consider a kinetic model which describes the orientation of microscopic rodlike particles. In this model $f(t, x, n) d n$ describes the time dependent probability that a rod with center of mass at the macroscopic position $x \in \mathbb{R}^{d}$ has at time $t \in \mathbb{R}^{+}$an axis in the area element $d n$. Here $n \in S^{d-1}$ is a director on the unit sphere embedded in $\mathbb{R}^{d}$. The physical application we are interested in assumes $d=3$. In order to verify our numerical methods we will also consider the case $d=2$.

We assume that the relations

$$
f \geq 0 \text { and } \int_{S^{d-1}} f d n=1
$$

hold for all time. The evolution of the distribution function $f=f(t, x, n)$ is described by the Smoluchowski equation

$$
\partial_{t} f+u \cdot \nabla_{x} f+\nabla_{n} \cdot\left(P_{n^{\perp}}, \nabla_{x} u n f\right)=D_{r} \Delta_{n} f+D \Delta_{x} f
$$

where $u: \mathbb{R}^{d} \times \mathbb{R}^{+} \rightarrow \mathbb{R}^{d}$ is a macroscopic velocity field which depends on $x$ and $t$. The second term on the left hand side describes the transport of the rods by the macroscopic velocity field $u$. The third term describes a rotation of the rod-like particles due to a macroscopic velocity gradient $\nabla_{x} u$. Here $P_{n^{\perp}}:=\nabla_{x}$ un $-\left(n \cdot \nabla_{x} u n\right) n$ denotes the projection of the vector $\nabla_{x}$ un on the tangent space in $n$. The terms on the right hand side model rotation and translation of the rod-like particles due to Brownian motion. Here $D_{r} \in \mathbb{R}^{+}$is the rotational diffusion constant and $D \in \mathbb{R}^{+}$is the translational diffusion constant.

A velocity gradient $\nabla_{x} u \neq 0$ distorts an isotropic distribution of $f$. This leads to an increase in entropy, which needs to be balanced (see $[3,12]$ ) by a stress tensor of the form

$$
\sigma(x, t):=\int_{S^{d-1}}(d n \otimes n-I) f d n .
$$

This stress tensor appears as additional term in the macroscopic flow equation. The macroscopic flow is described by the Stokes or Navier-Stokes equation, i.e.

$$
\begin{aligned}
& R e\left(\partial_{t} u+\left(u \cdot \nabla_{x}\right) u\right)=\mu \Delta_{x} u+\nabla_{x} p+\nabla_{x} \cdot \sigma, \\
& \nabla_{x} \cdot u=0 .
\end{aligned}
$$


Throughout this paper we denote with $\nabla_{x}, \nabla_{x}$. and $\Delta_{x}$ gradient, divergence and Laplacian in physical space. With $\nabla_{n}, \nabla_{n}$. and $\Delta_{n}$ we denote gradient, divergence and Laplacian on the sphere. Recently, Helzel and Tzavaras [9] have studied a modification of the above model (2.1)-(2.3), which describes the sedimentation in dilute suspensions of rod-like particles.

A resolved numerical method for the system (2.1)-(2.3) (or the model from [9]) requires, as a component, a solver for the Smoluchowski equation in the form

$$
\begin{aligned}
& \partial_{t} f(t, n)+\nabla_{n} \cdot\left(P_{n \perp} \nabla_{x} u_{e x t} n f(t, n)\right)=D_{r} \Delta_{n} f(t, n), \\
& f(0, n)=f_{0}(n),
\end{aligned}
$$

with $n \in S^{d-1}$ and $t \in \mathbb{R}^{+}$. This is the subject of our paper. The matrix $\nabla_{x} u_{e x t}$ now describes a constant externally imposed velocity gradient. (2.4) is a drift-diffusion equation on the sphere $S^{d-1}$. In the full model, this subproblem needs to be solved on every physical point of interested $x \in \mathbb{R}^{d}$ which is used in the discretization of the full model.

Most commonly, pdes on the sphere are solved by rewriting the problem in spherical coordinates. In order to rewrite (2.4), we introduce the notation

$$
n:=\left(\begin{array}{c}
\cos \theta \sin \phi \\
\sin \theta \sin \phi \\
-\cos \phi
\end{array}\right), \quad e_{\theta}:=\frac{1}{\sin \phi} \frac{\partial n}{\partial \theta}=\left(\begin{array}{c}
-\sin \theta \\
\cos \theta \\
0
\end{array}\right), \quad e_{\phi}:=\frac{\partial n}{\partial \phi}=\left(\begin{array}{c}
\cos \theta \cos \phi \\
\sin \theta \sin \phi \\
\sin \phi
\end{array}\right)
$$

with $\theta \in[0,2 \pi]$ and $\phi \in[0, \pi]$. Note that $\left\{n, e_{\theta}, e_{\phi}\right\}$ is an orthonormal basis of $\mathbb{R}^{3}$ and thus $\left\{e_{\theta}, e_{\phi}\right\}$ is an orthonormal basis of the tangent space of $S^{2}$ at $n$. We decompose the drift velocity $P_{n^{\perp}} \nabla_{x} u_{e x t} n$ using the basis of the tangent space and obtain

$$
P_{n} \nabla_{x} u_{e x t} n:=b_{\theta} e_{\theta}+b_{\phi} e_{\phi}
$$

with $b_{\theta}:=P_{n^{\perp}} \nabla_{x} u_{e x t} n \cdot e_{\theta}$ and $b_{\phi}:=P_{n^{\perp}} \nabla_{x} u_{e x t} n \cdot e_{\phi}$. Recall that for functions $f$ on $S^{2}$ the gradient operator can be expressed in the form

$$
\nabla_{n} f=\frac{1}{\sin \phi}\left(\partial_{\theta} f\right) e_{\theta}+\left(\partial_{\phi} f\right) e_{\phi}
$$

and the Laplace Beltrami operator can be expressed in the form

$$
\Delta_{n} f=\partial_{\theta}\left(\frac{1}{\sin \phi} \partial_{\theta} f\right)+\partial_{\phi}\left(\sin \phi \partial_{\phi} f\right) .
$$

Following Helzel and Otto [8], we can now rewrite (2.4) in the form

$$
\sin \phi \partial_{t} f+\partial_{\theta}\left(b_{\theta} f\right)+\partial_{\phi}\left(\sin \phi b_{\phi} f\right)=D_{r}\left(\partial_{\theta}\left(\frac{1}{\sin \phi} \partial_{\theta} f\right)+\partial_{\phi}\left(\sin \phi \partial_{\phi} f\right)\right) .
$$

In [8], a finite volume method was used in order to approximate the Smoluchowski equation in spherical coordinates. As can be seen from (2.5), singularities will arise at the poles $\phi=0$ and $\phi=\pi$, where $\sin \phi=0$. This requires a special numerical treatment.

The main goal of this paper is to introduce a numerical method for (2.4) which is based on the use of radial basis functions. This method does not suffer from a pole singularity. Furthermore, we will see that the construction of such methods is simpler than the construction of spectral methods, while we achieve a comparable accuracy. 
The RBF method should be of interest for a variety of applications. Note, for example, that modified versions of the Smoluchowski equation arise if we include more physical effects. For example, a modified rotational diffusion is used in the semi-dilute regime and a modified drift term on the sphere is used to model excluded volume effects in the concentrated regime [3]. Related kinetic models arise in the modelling of active spherical particles, see for example [1]. An active particle is described by its position and a velocity vector. This is very similar to a (passive) rod-like particle, which is described by its position and orientation.

\section{Numerical Methods for a Simplified Model}

In this section we consider the Smoluchowski equation on $S^{1}$ (i.e. on a circle), where Equation (2.4) simplifies to a one-dimensional transport-diffusion equation. We set $n=(\cos \theta, \sin \theta)^{T}$, $0 \leq \theta \leq 2 \pi$, choose $\nabla_{x} u_{e x t} \in \mathbb{R}^{2 \times 2}$ and obtain

$$
\nabla_{n} \cdot\left(P_{n \perp} \nabla_{x} u_{e x t} n f\right)=\partial_{\theta}\left(\left(\begin{array}{r}
-\sin \theta \\
\cos \theta
\end{array}\right) \cdot \nabla_{x} u_{e x t}\left(\begin{array}{c}
\cos \theta \\
\sin \theta
\end{array}\right) f\right) .
$$

Now we can consider different externally imposed velocity gradients $\nabla_{x} u_{e x t}$ and the corresponding drift terms (3.1). For shear flow we obtain

$$
\nabla_{x} u_{e x t}=\left(\begin{array}{cc}
0 & 0 \\
1 & 0
\end{array}\right), \quad P_{n^{\perp}} \nabla_{x} u_{e x t} n=\cos ^{2}(\theta)
$$

for elongational flow we obtain

$$
\nabla_{x} u_{e x t}=\left(\begin{array}{rr}
1 & 0 \\
0 & -1
\end{array}\right), \quad P_{n^{\perp}} \nabla_{x} u_{e x t} n=-\sin (2 \theta)
$$

and for rotational flow we obtain

$$
\nabla_{x} u_{e x t}=\left(\begin{array}{rr}
0 & 1 \\
-1 & 0
\end{array}\right), \quad P_{n \perp} \nabla_{x} u_{e x t} n=-1 .
$$

Note that multiplying the different matrices $\nabla_{x} u_{e x t}$ with a constant leads to an additional constant factor in the drift term. Later in this paper we will for example also consider shear flow with $v_{x} \neq 1$. Furthermore, we can consider shear flow with $u_{y} \neq 0$ and $u_{x}=v_{x}=v_{y}=0$.

In the general case we have

$$
\nabla_{x} u_{e x t}=\left(\begin{array}{cc}
u_{x} & u_{y} \\
v_{x} & v_{y}
\end{array}\right), \quad P_{n^{\perp}} \nabla_{x} u_{e x t} n=\left(v_{y}-u_{x}\right) \cos \theta \sin \theta-u_{y} \sin ^{2} \theta+v_{x} \cos ^{2} \theta .
$$

The Laplace-Beltrami operator on $S^{1}$ reduces to the standard second derivative. Thus, we obtain an advection-diffusion equations of the form

$$
\begin{aligned}
& \partial_{t} f(t, \theta)+\partial_{\theta}\left(\left(\left(v_{y}-u_{x}\right) \frac{1}{2} \sin 2 \theta-u_{y} \sin ^{2} \theta+v_{x} \cos ^{2} \theta\right) f(t, \theta)\right)=D_{r} \partial_{\theta \theta} f(t, \theta), \\
& f(\theta, 0)=f_{0}(\theta) .
\end{aligned}
$$

Note that (3.3) can also be obtained from (2.5) by setting $\phi=\pi / 2$ and $\partial_{\phi} f=0$. We impose the periodicity condition $f(t, 0)=f(t, 2 \pi)$ for all $t \geq 0$. The one-dimensional Equation (3.3) does of course not suffer from a pole singularity. 
For smooth solutions (3.3) can equivalently be expressed in the form

$$
\begin{aligned}
& \partial_{t} f(t, \theta)=\left(\left(u_{x}-v_{y}\right) \cos 2 \theta+\left(u_{y}+v_{x}\right) \sin 2 \theta\right) f(t, \theta) \\
& \quad+\left(\left(u_{x}-v_{y}\right) \frac{1}{2} \sin 2 \theta+u_{y} \sin ^{2} \theta-v_{x} \cos ^{2} \theta\right) \partial_{\theta} f(t, \theta) \\
& \quad+D_{r} \partial_{\theta \theta} f(t, \theta), \\
& f(\theta, 0)=f_{0}(\theta) .
\end{aligned}
$$

\subsection{Spectral method}

A spectral method for the one-dimensional Smoluchowski equation (3.3) is based on the ansatz

$$
f(t, \theta)=f_{0}(t)+\sum_{i=1}^{N} c_{i}(t) \cos (2 i \theta)+s_{i}(t) \sin (2 i \theta) .
$$

Inserting this ansatz into (3.3) and matching equal sine and cosine terms, leads to a linear system of ordinary differential equations of the general form $\mathbf{q}^{\prime}(t)=D \mathbf{q}(t)$ with the unknown coefficient vector $\mathbf{q}(t)=\left(f_{0}(t), c_{1}(t), s_{1}(t), \ldots, c_{N}(t), s_{N}(t)\right)^{T}$ and a $(2 N+1) \times(2 N+1)$ matrix $D$. We briefly illustrate this procedure for shear flow (i.e., we set $v_{x} \neq 0, u_{x}=u_{y}=v_{y}=0$ ) and $N=1$. In this case we obtain

$$
\begin{aligned}
& f(t, \theta) \approx f_{0}(t)+c_{1}(t) \cos 2 \theta+s_{1}(t) \sin 2 \theta \\
& \partial_{t} f(t, \theta) \approx f_{0}^{\prime}(t)+c_{1}^{\prime}(t) \cos 2 \theta+s_{1}^{\prime}(t) \sin 2 \theta \\
& \partial_{\theta} f(t, \theta) \approx-2 c_{1}(t) \sin 2 \theta+2 s_{1}(t) \cos 2 \theta \\
& \partial_{\theta \theta} f(t, \theta) \approx-4 c_{1}(t) \cos 2 \theta-4 s_{1}(t) \sin 2 \theta
\end{aligned}
$$

Now we insert these terms into the Smoluchowski equation for shear flow, i.e. into the pde

$$
\partial_{t} f+\partial_{\theta}\left(v_{x} \cos ^{2} \theta f\right)=D_{r} \partial_{\theta \theta} f .
$$

After some straight forward calculations we obtain

$$
\begin{aligned}
& f_{0}^{\prime}(t)+c_{1}^{\prime}(t) \cos 2 \theta+s_{1}^{\prime}(t) \sin 2 \theta+v_{x}\left(s_{1} \cos 2 \theta-c_{1} \sin 2 \theta\right. \\
& \left.\quad-f_{0}(t) \sin 2 \theta+s_{1} \cos 4 \theta-c_{1} \sin 4 \theta\right) \\
= & D_{r}\left(-4 c_{1} \cos 2 \theta-4 s_{1} \sin 2 \theta\right) .
\end{aligned}
$$

Now we group terms of different order in $\cos 2 i \theta$ and $\sin 2 i \theta$. In our simple situation we obtain

$$
\begin{aligned}
1: & f_{0}^{\prime}(t)=0 \\
\cos 2 \theta: & c_{1}^{\prime}(t)+v_{x} s_{1}(t)=-4 D_{r} c_{1}(t) \\
\sin 2 \theta: & s_{1}^{\prime}(t)-v_{x} c_{1}(t)-v_{x} f_{0}(t)=-4 D_{r} s_{1}(t) .
\end{aligned}
$$

Terms of higher order will be ignored (for $N=1$ ). This leads to the ordinary differential equation

$$
\left(\begin{array}{c}
f_{0}(t) \\
c_{1}(t) \\
s_{1}(t)
\end{array}\right)^{\prime}=\left(\begin{array}{ccc}
0 & 0 & 0 \\
0 & -4 D_{r} & -v_{x} \\
v_{x} & v_{x} & -4 D_{r}
\end{array}\right)\left(\begin{array}{l}
f_{0}(t) \\
c_{1}(t) \\
s_{1}(t)
\end{array}\right)
$$


In order to describe the ode for general $N$ and the general velocity gradient described in (3.2), let $D_{1}$ denote the $N \times N$ diagonal matrix with entries $d_{i i}:=-4 i^{2}, i=1, \ldots, N$. Then the second order derivative term contributes to the matrix $D$ with entries of the form $D_{r} D_{1} \otimes\left(\begin{array}{ll}1 & 0 \\ 0 & 1\end{array}\right)$. As we can already see from our simple example, these $2 N$ terms do not contribute to the first row and the first column of $D$. To indicate this we write

$$
D(2: 2 N+1,2: 2 N+1)=D_{r} D_{1} \otimes\left(\begin{array}{ll}
1 & 0 \\
0 & 1
\end{array}\right) .
$$

In order to define the contributions of the first derivative terms to the matrix $D$, we define $D_{2}$ to be the $N \times N$ diagonal matrix with components $d_{i i}:=i, i=1, \ldots, N$. Furthermore, $D_{3}$ is the $N \times N$ matrix with components $d_{i, i+1}:=i / 2$ for $i=1, \ldots, N-1$ and all other entries equal to zero. Finally, $D_{4}$ is the $N \times N$ matrix with entries $d_{i+1, i}:=(i+1) / 2, i=1, \ldots, N-1$ and all other entries equal to zero. With these definitions the contributions of $D(2: 2 N+1,2: 2 N+1)$, which are caused by the drift term, can be expressed in the form

$D_{2} \otimes\left(\begin{array}{cc}0 & -u_{y}+v_{x} \\ u_{y}-v_{x} & 0\end{array}\right)+D_{3} \otimes\left(\begin{array}{cc}-u_{x}+v_{y} & -u_{y}-v_{x} \\ u_{y}+v_{x} & -u_{x}+v_{y}\end{array}\right)+D_{4} \otimes\left(\begin{array}{cc}u_{x}-v_{y} & -u_{y}-v_{x} \\ u_{y}+v_{x} & u_{x}-v_{y}\end{array}\right)$.

Furthermore, the first column of $D$ contains non-zero entries in the second and third row. They have the form $D(2,1):=u_{x}-v_{y}$ and $D(3,1):=v_{x}+u_{y}$.

We used the ode45 routine from MATLAB in order to solve the ordinary differential equation $\mathbf{q}^{\prime}(t)=D \mathbf{q}(t)$. Note that the derivation needs to be performed for each partial differential equation. Numerical results obtained with this method will be shown in Section 3.3.

\subsection{RBF method}

The approximation of (3.3) using radial basis functions is based on an interpolation of the solution $f$ which we denote by $\tilde{f}$ and which takes the form

$$
\tilde{f}(t, \theta)=\sum_{j=1}^{M} c_{j}(t) \Phi\left(\left\|n-n_{j}\right\|_{2}, \epsilon\right) .
$$

Here, $n \in S^{1}$ on the right hand side of $(3.5)$ is related to $\theta$ via the relation $n=\left(\begin{array}{c}\cos \theta \\ \sin \theta\end{array}\right),\|\cdot\|_{2}$ is the standard Euclidean vector norm. We assign points $n_{1}, \ldots, n_{M}$ on $S^{1}$ using equally distribute points $\theta_{i}:=i \Delta \theta, \Delta \theta=2 \pi / M, i=1, \ldots, M$. Distances between points on $S^{1}$ in $\|\cdot\|_{2}$ can now easily be computed, which automatically imposes the periodicity condition. The function $\Phi$ is a so-called radial basis function. It depends on a distance function $r=r(n)=\left\|n-n_{j}\right\|_{2}$ as well as on a shape parameter $\epsilon$. Different choices of radial basis functions have been discussed in Fornberg and Piret [7]. Below we will sometimes use the notation $\Phi_{j}(r)$, which for simplicity omits the dependence on $\epsilon$ and which indicate that the radial basis function is centered at $n_{j}$. For our numerical computations we use the Inverse Multiquadric (IMQ) RBF, which has the form

$$
\Phi(r, \epsilon)=1 / \sqrt{1+\epsilon^{2} r^{2}}
$$

The parameter $\epsilon$ controls the shape of the RBF and in turn the accuracy of the solution as discussed in detail in [7]. 
The initial values for $f$ are used to calculate initial values for $c_{1}, \ldots, c_{M}$ using the interpolation condition

$$
\tilde{f}\left(0, \theta_{j}\right)=f\left(0, \theta_{j}\right), \quad j=1, \ldots, M .
$$

We can express this interpolation condition in the form of a linear system

$$
B \mathbf{c}(0)=\tilde{\mathbf{f}}(0),
$$

with vector valued quantities $\mathbf{c}(0)=\left(c_{1}(0), \ldots, c_{M}(0)\right)^{T}$ and $\tilde{\mathbf{f}}(0)=\left(f\left(0, \theta_{1}\right), \ldots, f\left(0, \theta_{M}\right)\right)^{T}$ and a matrix $B \in \mathbb{R}^{M \times M}$ with components $b_{i j}:=\Phi\left(\left\|n_{i}-n_{j}\right\|_{2}, \epsilon\right), i, j=1, \ldots, M$. Micchelli [11] showed that the matrix $B$ is positiv definite for typically used radial basis functions and any choice of pairwise distinct nodes.

Our goal is to compute $\tilde{\mathbf{f}}(t):=\left(\tilde{f}\left(t, \theta_{1}\right), \ldots, \tilde{f}\left(t, \theta_{M}\right)\right)^{T}$ using a method of lines approach. For a given vector $\tilde{\mathbf{f}}(t)$ we can recover the function $\tilde{f}(t, \theta)$ for all $0 \leq \theta \leq 2 \pi$, by solving a linear system of the form

$$
B \mathbf{c}(t)=\tilde{\mathbf{f}}(t)
$$

and subsequently using Equation (3.5). We also introduce the vector valued quantities

$$
\begin{aligned}
& \theta:=\left(\theta_{1}, \ldots, \theta_{M}\right)^{T}, \\
& \mathbf{f}_{t}(t):=\left(\partial_{t} \tilde{f}\left(t, \theta_{1}\right), \ldots, \partial_{t} \tilde{f}\left(t, \theta_{M}\right)\right)^{T}, \\
& \tilde{\mathbf{f}}_{\theta}(t):=\left(\left.\partial_{\theta} \tilde{f}(t, \theta)\right|_{\theta=\theta_{1}}, \ldots,\left.\partial_{\theta} \tilde{f}(t, \theta)\right|_{\theta=\theta_{M}}\right)^{T}, \\
& \tilde{\mathbf{f}}_{\theta \theta}(t):=\left(\left.\partial_{\theta \theta} \tilde{f}(t, \theta)\right|_{\theta=\theta_{1}}, \ldots,\left.\partial_{\theta \theta} \tilde{f}(t, \theta)\right|_{\theta=\theta_{M}}\right)^{T} .
\end{aligned}
$$

We will now describe the derivation of the differentiation matrices, which is the crucial step in the definition of the RBF method. Differentiation of (3.5) with respect to $\theta$ leads to

$$
\partial_{\theta} \tilde{f}(t, \theta)=\sum_{j=1}^{M} c_{j}(t) \frac{\partial \Phi_{j}(r)}{\partial r} \frac{\partial r}{\partial \theta} .
$$

Thus, the computation of the vector $\tilde{\mathbf{f}}_{\theta}(t)$ can be expressed in the form

$$
\tilde{\mathbf{f}}_{\theta}(t)=H_{1} \mathbf{c}(t),
$$

with a matrix $H_{1} \in \mathbb{R}^{M \times M}$ with entries

$$
h_{i j}:=\left.\frac{\partial \Phi_{j}(r)}{\partial r} \frac{\partial r}{\partial \theta}\right|_{\theta=\theta_{i}}, \quad i, j=1, \ldots, M .
$$

For the IMQ-RBF (3.6), for example, we get

$$
h_{i j}=-\frac{\epsilon^{2} \sin \left(\theta_{i}-\theta_{j}\right)}{\left(1+\epsilon^{2}\left(2-2 \cos \left(\theta_{i}-\theta_{j}\right)\right)\right)^{3 / 2}}, \quad i, j=1, \ldots, M .
$$

Combining (3.9) and (3.8) we obtain

$$
\tilde{\mathbf{f}}_{\theta}(t)=H_{1} B^{-1} \tilde{\mathbf{f}}(t) .
$$

The matrix $D_{1}:=H_{1} B^{-1}$ (in MATLAB H/B) is called the differentiation matrix for the first derivative with respect to $\theta$. In analogy we can express the discretization of the second derivative with respect to $\theta$ in the form

$$
\tilde{\mathbf{f}}_{\theta \theta}(t)=H_{2} \mathbf{c}(t)
$$


with a matrix $H_{2} \in \mathbb{R}^{M \times M}$ with entries

$$
h_{i j}=\left.\frac{\partial^{2} \Phi_{j}}{\partial \theta^{2}}\right|_{\theta=\theta_{i}}=\left.\left(\frac{\partial^{2} \Phi_{j}}{\partial r^{2}}\left(\frac{\partial r}{\partial \theta}\right)^{2}+\frac{\partial \Phi_{j}}{\partial r} \frac{\partial^{2} r}{\partial \theta^{2}}\right)\right|_{\theta=\theta_{i}} .
$$

For the IMQ-RBF we obtain

$$
\begin{aligned}
h_{i j}= & \frac{3 \epsilon^{4} \sin ^{2}\left(\theta_{i}-\theta_{j}\right)}{\left(1+\epsilon^{2}\left(\left(\cos \theta_{i}-\cos \theta_{j}\right)^{2}+\left(\sin \theta_{i}-\sin \theta_{j}\right)^{2}\right)\right)^{5 / 2}} \\
& -\frac{-\epsilon^{2} \cos \left(\theta_{i}-\theta_{j}\right)}{\left(1+\epsilon^{2}\left(\left(\cos \theta_{i}-\cos \theta_{j}\right)^{2}+\left(\sin \theta_{i}-\sin \theta_{j}\right)^{2}\right)\right)^{3 / 2}} .
\end{aligned}
$$

Using (3.8) and (3.11), we can express the second derivative in the form

$$
\tilde{\mathbf{f}}_{\theta \theta}(t)=H_{2} B^{-1} \tilde{\mathbf{f}}(t) .
$$

The matrix $D_{2}:=H_{2} B^{-1}$ is called the differentiation matrix for the second derivative.

On the discrete level, the pde (3.3) with the drift term (3.2) can be expressed in the form of an ordinary differential equation

$$
\begin{aligned}
\tilde{\mathbf{f}}_{t}(t)=( & \left.\left(u_{x}-v_{y}\right) \operatorname{diag}(\cos 2 \boldsymbol{\theta})+\left(u_{y}+v_{x}\right) \operatorname{diag}(\sin 2 \boldsymbol{\theta})\right) \tilde{\boldsymbol{f}}(t) \\
& +\left(u_{y} \operatorname{diag}\left(\sin ^{2} \boldsymbol{\theta}\right)-v_{x} \operatorname{diag}\left(\cos ^{2} \boldsymbol{\theta}\right)+\frac{1}{2}\left(u_{x}-v_{y}\right) \operatorname{diag}(\sin 2 \boldsymbol{\theta})\right) D_{1} \tilde{\mathbf{f}}(t) \\
& +D_{r} D_{2} \tilde{\mathbf{f}}(t) .
\end{aligned}
$$

Here $\operatorname{diag}(\cos 2 \boldsymbol{\theta})$ is the diagonal matrix with $(i, i)$ component $\cos \left(2 \theta_{i}\right)$ and analogously for the other diagonal matrices. We use the MATLAB routine ode45 in order to solve (3.13) numerically. Note that we solve an ode for $\tilde{\mathbf{f}}(t)$ and not for $\mathbf{c}(t)$. The vector $\mathbf{c}$ only needs to be computed if we wish to evaluate $\tilde{f}$ at other points than the nodes, for example for plotting reasons.

Note furthermore, that once the differentiation matrices $D_{1}$ and $D_{2}$ are defined, the derivation of the system of ordinary differential equations (which needs to be solved in order to obtain the RBF method) is trivial. It is a straight forward approximation of (3.4). This is a main advantage of the RBF method compared to the spectral method. In the next section we will see that the accuracy of the RBF method is comparable with the accuracy of the spectral method.

\subsection{Numerical results}

We first consider steady state calculations of the Smoluchowski equation for elongational flow.

Example 3.1. We consider on $S^{1}$ the partial differential equation

$$
\partial_{t} f+\partial_{\theta}\left(-\frac{3}{2} \sin (2 \theta) f\right)=D_{r} \partial_{\theta \theta} f
$$

with initial values $f(0, \theta)=1 /(2 \pi)$. This problem has a steady state solution of the form

$$
f_{e q}(\theta)=C \exp \left(-\frac{3}{2 D_{r}} \sin ^{2} \theta\right)
$$

with the constant $C$ such that $\int_{0}^{2 \pi} f_{e q}(\theta) d \theta=\int_{0}^{2 \pi} f(0, \theta) d \theta=1$. 
In Fig. 3.1, we show the steady state solution of Example 3.1 plotted over $S^{1}$ for two different values of $D_{r}$. Note that we approximate this time dependent problem for a time which is large enough so that the solution reaches the steady state. In the diffusion dominated regime, shown in the left plot, there is only a slight preference of the horizontal direction. In the transport dominated case, shown in the right plot, the rods orient strongly in horizontal direction. In Fig. 3.2 , we show the max-norm error vs. $N$ for steady state solutions. In the diffusion dominated case, shown in the left plot, very accurate results can already be obtained with small values of $N$. The number of $N$ which is needed in order to obtain accurate approximations of the steady state solution increases as we decrease $D_{r}$, as can be seen in the middle and right plot. Note that for a fixed drift term, decreasing $D_{r}$ leads to a more transport dominated problem.
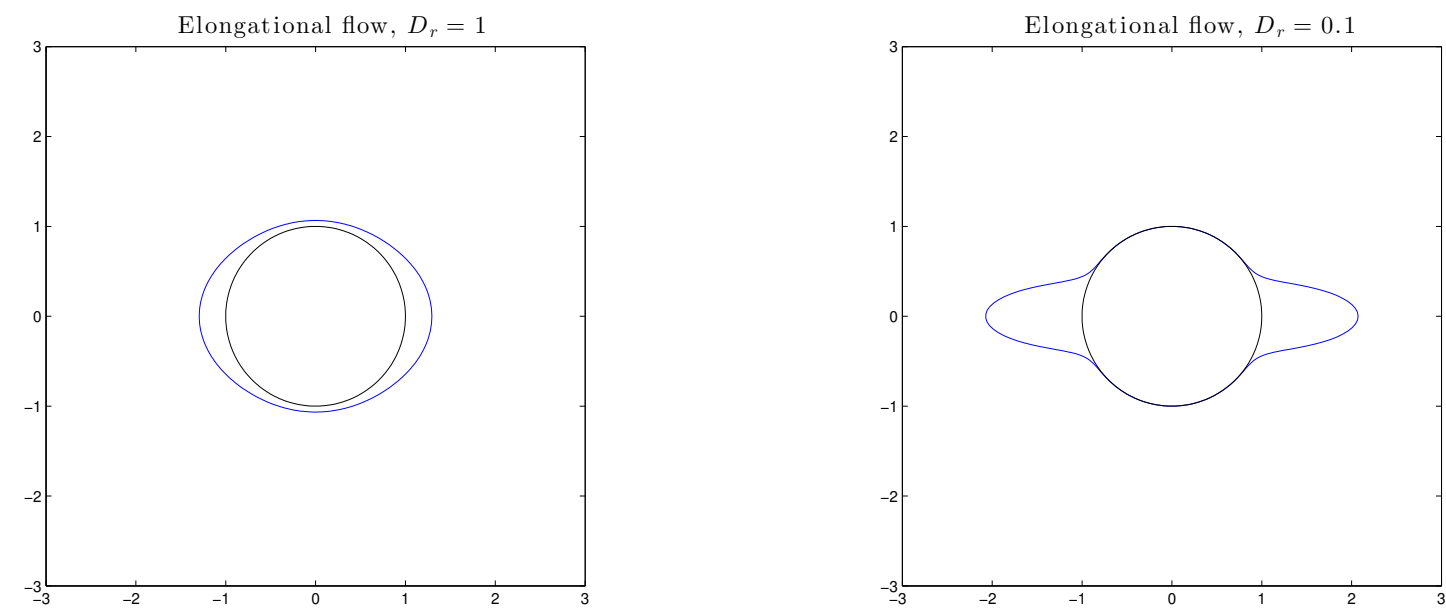

Fig. 3.1. Solution structure of elongational flow problem for two different values of $D_{r}$.
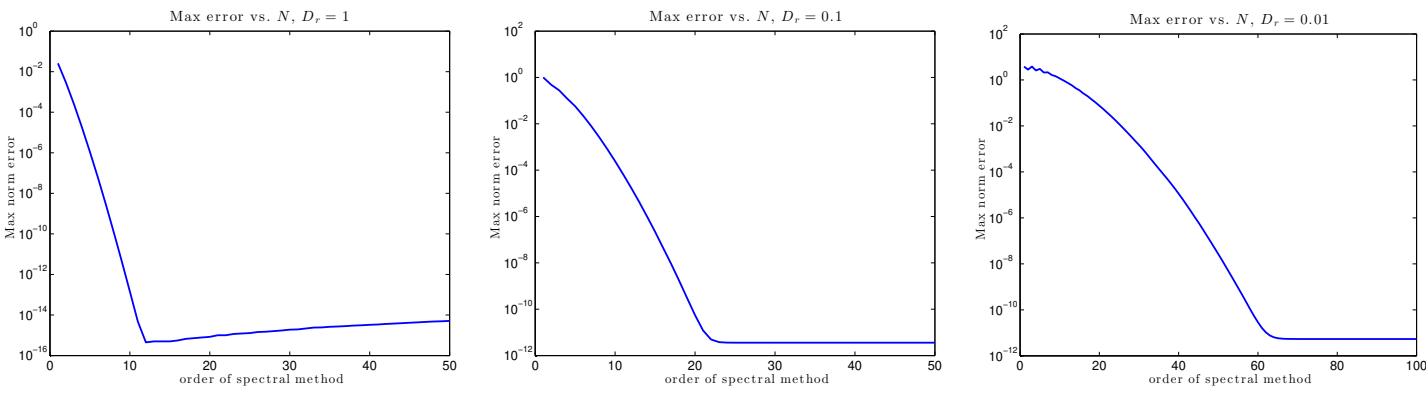

Fig. 3.2. Max-norm error vs. $N$ for steady state computations of elongational flow using the spectral method described in Section 3.1.

In Figs. 3.3-3.4 we show plots of the error vs. $M$ for computations obtained with the RBF method. In the diffusion dominated case, less points are needed to resolve the solution structure. In the convection dominated regime, more points are required. Furthermore, the accuracy of the solution depends on the choice of $\epsilon$. Smaller values of $\epsilon$ are more accurate provided that the resulting method is stable. For $D_{r}=0.1$ and $\epsilon=1$, for example, the method became unstable 

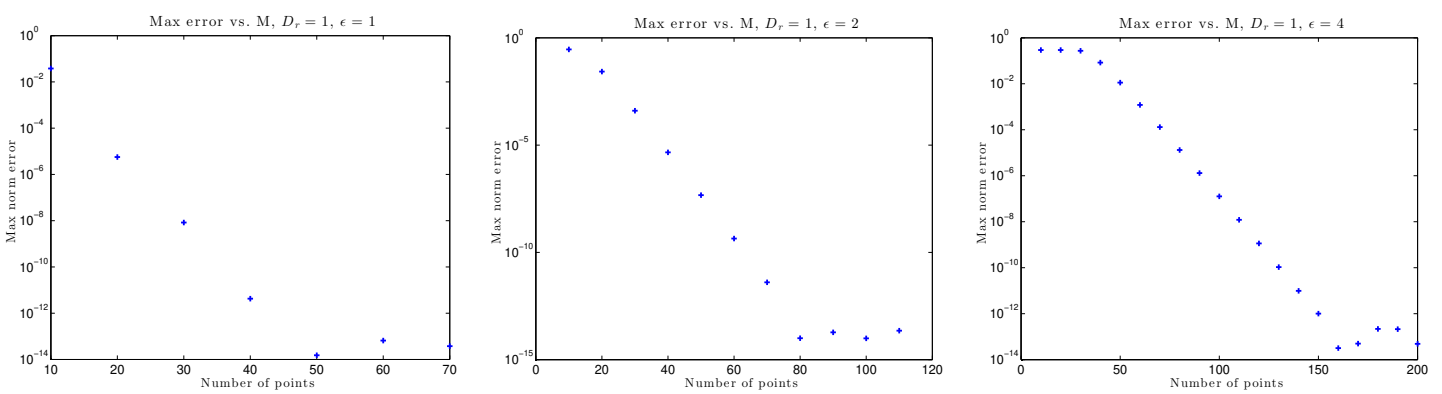

Fig. 3.3. Max-norm error vs. $M$ for steady state computations of elongational flow using the RBF method described in Section 3.2 with $D_{r}=1$ and (left plot) $\epsilon=1$, (middle) $\epsilon=2$ and (right) $\epsilon=4$.
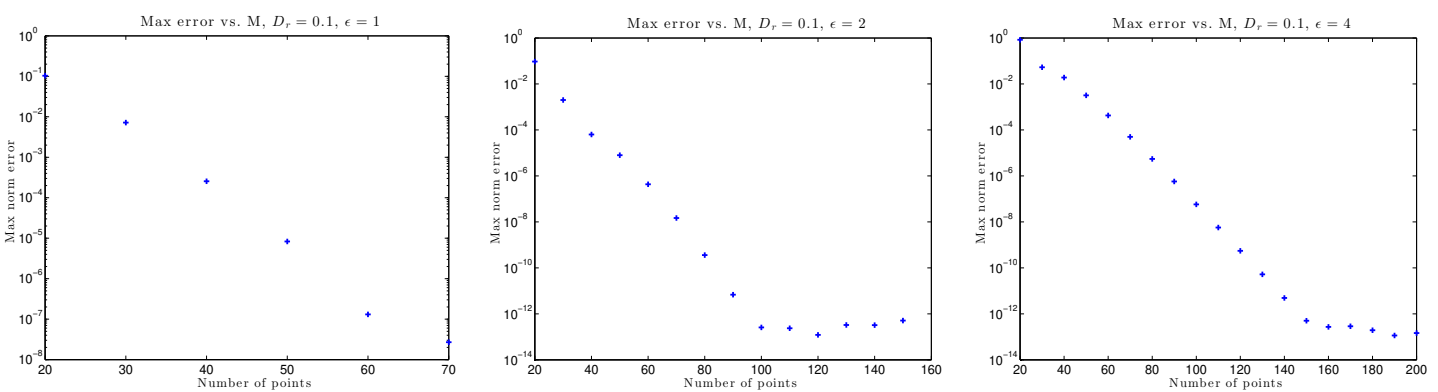

Fig. 3.4. Max-norm error vs. $M$ for steady state computations of elongational flow using the RBF method described in Section 3.2 with $D_{r}=0.1$ and (left plot) $\epsilon=1$, (middle) $\epsilon=2$ and (right) $\epsilon=4$.

for larger values of $M$. Both, with the spectral method but also with the RBF method we can reach an accuracy which is on the order of magnitude of the machine precision.

In our next example we consider steady state approximations of the Smoluchowski equation for an externally imposed shear flow.

Example 3.2. We consider on $S^{1}$ the initial value problem

$$
\begin{aligned}
& \partial_{t} f+\partial_{\theta}\left(v_{x} \cos ^{2} \theta f\right)=D_{r} \partial_{\theta \theta} f \\
& f(0, \theta)=1 / 2 \pi .
\end{aligned}
$$

In Fig. 3.5, we present steady state solutions of Example 3.2 for different values of $v_{x} / D_{r}$. We used the spectral method in order to calculate these solutions.

In Figs. 3.6 and 3.7 we compare the solution structure of Example 3.2 obtained by using the RBF method (red circles) on different grids with a highly resolved spectral method (solid line). We show results for $v_{x} / D_{r}=100$ and $v_{x} / D_{r}=1000$ and used different grid resolutions. In most of the calculations we set $\epsilon=4$. However, in the case of strong shear rate, the coarse grid computation using $M=50$ produced oscillations for $\epsilon=4$. These are caused by the poor resolution of the peaked solution structure. By using a larger value of $\epsilon$, those oscillations where attenuated. Here we show a plot for which we used $\epsilon=8$. Our numerical results show that the RBF method produces very accurate results, provided that the solution structure is sufficiently resolved. Compared to other methods, such as finite difference or finite volume methods, the 

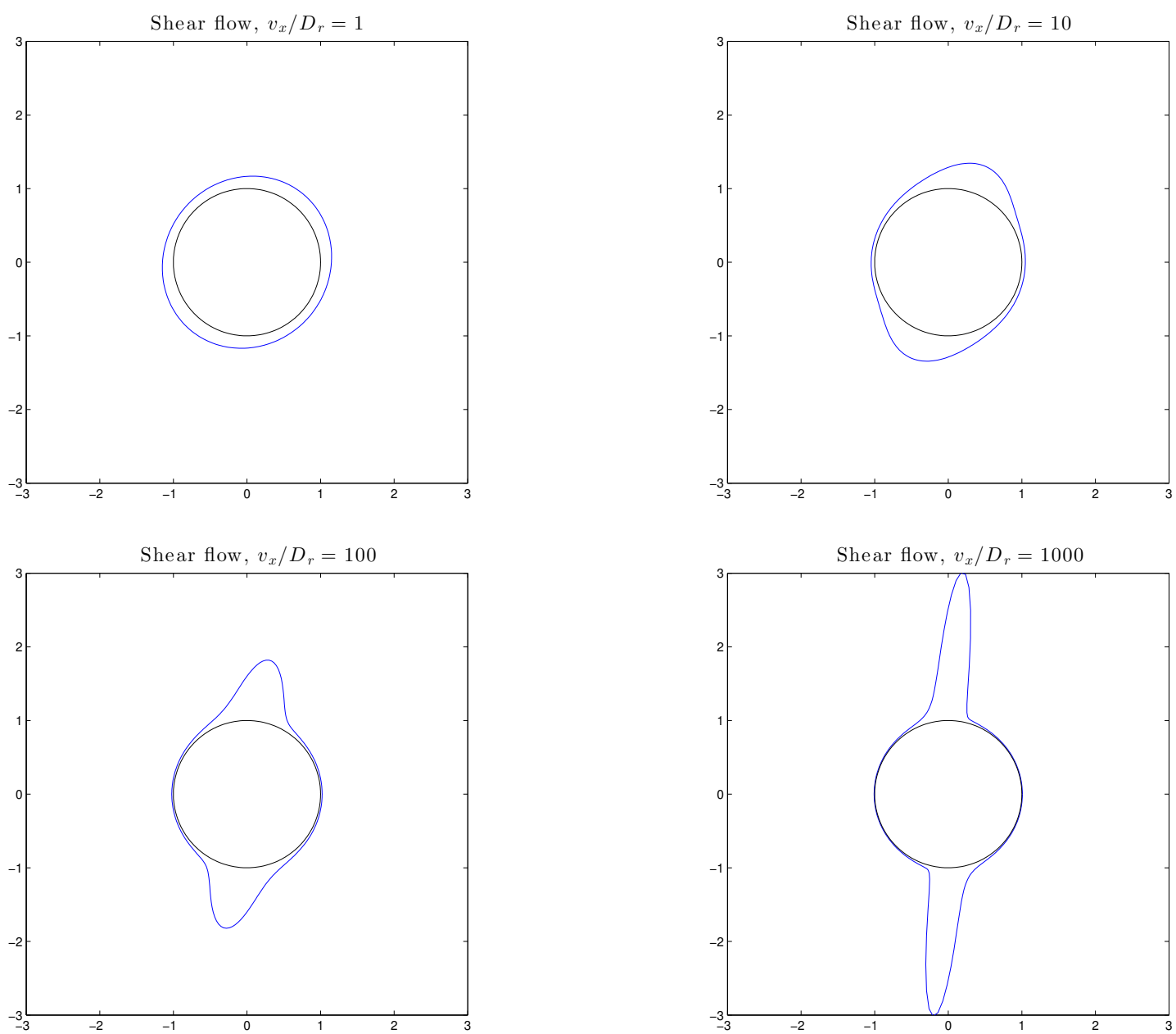

Fig. 3.5. Solution structure of shear flow for different values of $v_{x} / D_{r}$.
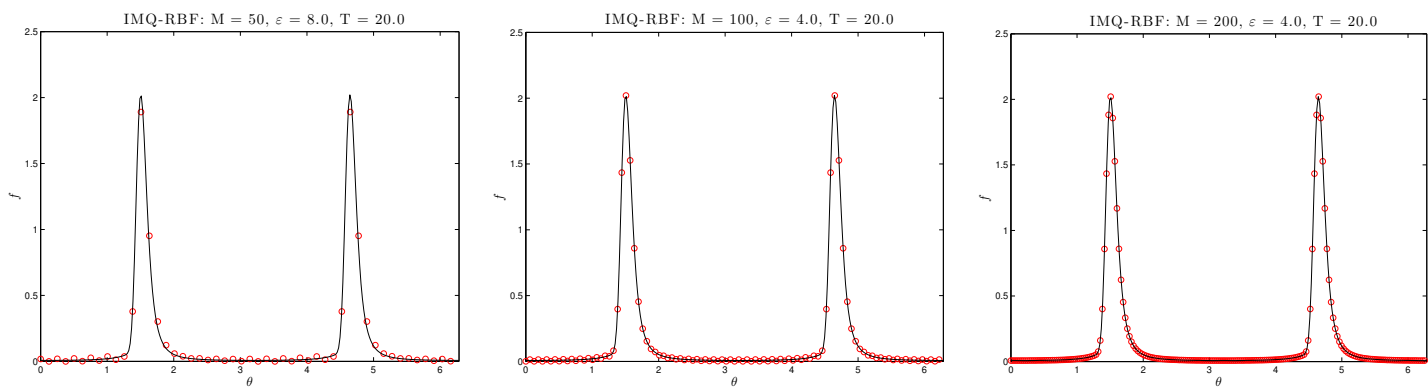

Fig. 3.6. Computation of the shear flow problem with $v_{x} / D_{r}=100$ using a highly resolved spectral method (solid line) as well as the RBF method (red circles). For the RBF computation we used 50,100 and 200 node points.

resolution which was needed is relatively coarse. Thus, the RBF method is an efficient method for these kind of problems. 

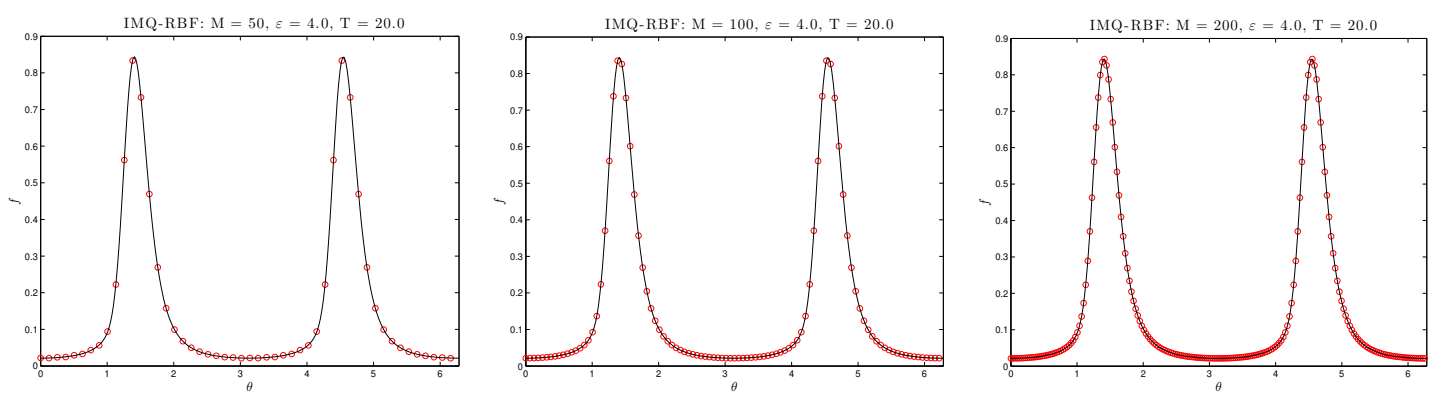

Fig. 3.7. Computation of the shear flow problem with $v_{x} / D_{r}=1000$ using a highly resolved spectral method (solid line) as well as the RBF method (red circles). For the RBF computation we used 50, 100 and 200 node points.

\section{The RBF Method on $S^{2}$}

In this section we describe an RBF method for the Smoluchowski equation on $S^{2}$. This method is again based on an interpolation of the solution using radial basis functions, which can be expressed in the form

$$
\tilde{f}(t, n)=\sum_{j=1}^{M} c_{j}(t) \Phi\left(\left\|n-n_{j}\right\|_{2}, \epsilon\right),
$$

with $n \in S^{2}$, i.e. $n \in \mathbb{R}^{3}$ and $\|n\|_{2}=1$. Now $n_{1}, \ldots, n_{M}$ are points on $S^{2}$. Here we used the so-called maximal determinant (MD) nodes as well as the minimal energy (ME) nodes, which can be downloaded from [15]. In analogy to the one-dimensional case, the coefficient vector $\mathbf{c}(t)=\left(c_{1}(t), \ldots, c_{M}(t)\right)^{T}$ is related to the vector $\tilde{\mathbf{f}}(t)=\left(\tilde{f}\left(t, n_{1}\right), \ldots, \tilde{f}\left(t, n_{M}\right)\right)^{T}$ via a matrix vector multiplication of the form

$$
B \mathbf{c}(t)=\tilde{\mathbf{f}}(t)
$$

with $B \in \mathbb{R}^{M \times M}$ and $b_{i j}:=\Phi\left(\left\|n_{i}-n_{j}\right\|_{2}, \epsilon\right), i, j=1, \ldots, M$.

In order to discretize the spatial derivatives, the method makes use of the fact that the gradient operator on the sphere $\nabla_{n}$ is related to the gradient operator in Euclidian space $\nabla$ through

$$
\nabla_{n}=(I d-n \otimes n) \cdot \nabla,
$$

with $n \in S^{2}$. Let $\Phi_{k}(r(n))$ denote the RBF centered at $n_{k}$, with $r(n):=\left\|n-n_{j}\right\|_{2}$. Using the chain rule, the gradient in Cartesian space of $\Phi_{k}(r(x))$ is given by

$$
\nabla \Phi_{k}(r(n))=\left(n-n_{k}\right) \frac{\Phi_{k}^{\prime}(r(n))}{r(n)} .
$$

Consequently, the gradient on the sphere of the continuous function $\Phi_{k}(r(n))$ can be expressed by

$$
\nabla_{n} \Phi_{k}(r(n))=(I d-n \otimes n)\left(n-n_{k}\right) \frac{\Phi_{k}^{\prime}(r(n))}{r(n)} .
$$

In our numerical method we want to express the components of the gradient at all of the discretization points $n_{i}, i=1, \ldots, M$ at once. This can be done by three matrix vector multiplications with $M \times M$ matrices, one for each component of the gradient in (4.3). We denote 
the differentiation matrices for the first, second and third component of (4.3) by $D_{N}^{x}, D_{N}^{y}, D_{N}^{z}$, respectively. In order to describe these matrices, we use the notation $n=(x, y, z)^{T}$ for the components of the vector $n$. We obtain

$$
D_{N}^{x}:=D^{x} B^{-1}, \quad D_{N}^{y}:=D^{y} B^{-1}, \quad D_{N}^{z}:=D^{z} B^{-1}
$$

with a matrix $D^{x}$ that has the components

$$
d_{j k}^{x}:=\left(x_{j} n_{j}^{T} n_{k}-x_{k}\right) \frac{\Phi_{k}^{\prime}\left(r\left(n_{j}\right)\right)}{r\left(n_{j}\right)}, \quad j, k=1, \ldots, M .
$$

Note that $n_{j}$ and $n_{k}$ are vectors, while $x_{j}$ is the first component of $n_{j}$. In analogy the matrix $D^{y}$ has the components

$$
d_{j k}^{y}:=\left(y_{j} n_{j}^{T} n_{k}-y_{k}\right) \frac{\Phi_{k}^{\prime}\left(r\left(n_{j}\right)\right)}{r\left(n_{j}\right)}
$$

and the matrix $D^{z}$ has the components

$$
d_{j k}^{z}:=\left(z_{j} n_{j}^{T} n_{k}-z_{k}\right) \frac{\Phi_{k}^{\prime}\left(r\left(n_{j}\right)\right)}{r\left(n_{j}\right)} .
$$

From the structure of the radial basis function (compare with (3.6)) it follows that the first derivative $\Phi^{\prime}(r)$ contains a factor $r$ in the enumerator. This factor cancels with the $r$ in the denominator. Therefore, the division by $r$ in the above formulas does not cause any stability problems.

The RBF discretization of the Laplace Beltrami operator was introduced in [16]. In order to describe this approach, let $d(n):=\left\|n-n_{r e f}\right\|_{2}$ denote the Euclidian distance of the point $n \in S^{2}$ from a reference point $n_{r e f}$, for example the north pole $n_{r e f}:=(0,0,1)$. Following [16], the Laplace Beltrami operator of a function $f(d)$ can be expressed in the form

$$
\Delta_{n} f(d)=\frac{1}{4}\left(\left(4-d^{2}\right) \frac{\partial^{2} f}{\partial d^{2}}+\frac{4-3 d^{2}}{d} \frac{\partial f}{\partial d}\right)
$$

The discretization of $\Delta_{n} f$ can again be described in the form of a matrix vector multiplication, with an $N \times N$ matrix of the form

$$
D_{L B}=D_{2} B^{-1}
$$

with $B$ as described above and a matrix $D_{2}$, which evaluates (4.4) at the discrete points $n_{1}, \ldots, n_{M}$. A crucial point to note is that (4.4) is independent of the choice of $n_{\text {ref }}$. In particular, we can chose $n_{r e f}=n_{i}$ for the discretization of the Laplace Beltrami operator at the point $n_{i}$. With this choice, the components of $D_{2}$ are given by

$$
d_{i j}:=\left(1-\frac{3}{4}\left\|n_{i}-n_{j}\right\|^{2}\right) \frac{\Phi_{j}^{\prime}\left(\left\|n_{i}-n_{j}\right\|\right)}{\left\|n_{i}-n_{j}\right\|}+\left(1-\frac{1}{4}\left\|n_{i}-n_{j}\right\|^{2}\right) \Phi_{j}^{\prime \prime}\left(\left\|n_{i}-n_{j}\right\|\right)
$$

for $i, j=1, \ldots, M$. The term $\left\|n_{i}-n_{j}\right\|$ in the denominator again cancels and therefore does not lead to stability problems.

Finally, we are ready to describe the discretization of (2.4) on $S^{2}$. Note that the drift term $P_{n} \perp \nabla_{x} u_{e x t} n$ is a vector valued quantity, which is defined on each point of $S^{2}$. We will refer to the three components of the drift in Cartesian coordinates separately. Therefore, we introduce the notation $P_{n^{\perp}} \nabla_{x} u_{e x t} n:=(u(n), v(n), w(n))^{T}$. Let $U, V, W$ describe $N \times N$ diagonal matrices with entries $u_{i i}:=u\left(n_{i}\right), v_{i i}:=v\left(n_{i}\right)$ and $w_{i i}:=w\left(n_{i}\right)$ for $i=1, \ldots, M$. In semi-discrete 
form, the RBF method for the Smoluchowski equation can now be described by the system of ordinary differential equations

$$
\tilde{\mathbf{f}}_{t}(t)=-\left(U D_{x}+V D_{y}+W D_{z}\right) \tilde{\mathbf{f}}(t)-\left(D_{x} U+D_{y} V+D_{z} W\right) \tilde{\mathbf{f}}(t)+D_{r} D_{L B} \tilde{\mathbf{f}}(t) .
$$

This system can now easily be solved by an explicit ode solver.

\subsection{Numerical results}

For our accuracy study, we again choose $\nabla_{x} u_{e x t} \in \mathbb{R}^{3 \times 3}$ in such a way that steady state solutions of (2.4) are known. This allows us to compare the numerical solution with the exact steady state solution. In our first example on $S^{2}$ we consider the Smoluchowski equation for elongational flow.

Example 4.1. We consider the initial value problem

$$
\begin{aligned}
& \partial_{t} f(t, n)+\nabla_{n} \cdot\left(P_{n^{\perp}} \nabla_{x} u_{e x t} n f(t, n)\right)=D_{r} \Delta_{n} f(t, n), \\
& f(0, n)=1 / 4 \pi,
\end{aligned}
$$

with $n \in S^{2}$ and an externally imposed velocity gradient which describes elongational flow, i.e.

$$
\nabla_{x} u_{e x t}:=\ell\left(\begin{array}{ccc}
2 & 0 & 0 \\
0 & -1 & 0 \\
0 & 0 & -1
\end{array}\right),
$$

with $\ell= \pm 1$. For $\ell>0$, we obtain uniaxial extensional motion, i.e., a full alignment of the director in one direction. For $\ell<0$ we observe biaxial extensional motion, i.e. the axes align strongly in a plane, but within that plane show no further tendency to align.

In spherical coordinates $\phi \in[0, \pi], \theta \in[0,2 \pi]$, the exact steady state solution can be expressed in the form

$$
\begin{array}{ll}
f_{e x}(\phi, \theta)=C_{1} \exp \left(-\frac{3}{2 D_{r}}\left(1-\cos ^{2}(\phi) \sin ^{2}(\theta)\right)\right) & \text { for } \ell=1, \\
f_{e x}(\phi, \theta)=C_{2} \exp \left(-\frac{3}{2 D_{r}} \cos ^{2}(\phi) \sin ^{2}(\theta)\right) & \text { for } \ell=-1 .
\end{array}
$$

The constants $C_{1}$ and $C_{2}$ respectively are obtained by the condition $\int_{S^{2}} f d n=1$. If we set the rotational diffusion constant to $D_{r}=0.1$, then we obtain $C_{1}=2.30121392$ and $C_{2}=$ 0.34776898 .

In Fig. 4.1 we show images of the exact steady state solution for Example 4.1. In order to test the algorithm we start with constant initial values $f(n, 0)=1 /(4 \pi)$ and evolve Equation (2.4) until a steady state solution is reached. In Figs. 4.2-4.3, we show the max norm error versus $M$ for steady state computations of uniaxial and biaxial extensional motion. For all computations we used $\epsilon=2.6$ and computed the numerical solution at time $T=10$. The max-norm error is obtained by comparing the vector $\tilde{\mathbf{f}}(T)$ with the exact steady state solution evaluated at the same nodes. Our computations show that we can again reach a good accuracy with relatively few degrees of freedom. The ME nodes (red circles) produced slightly more accurate results than the MD nodes (blue crosses). The accuracy is limited, since the system (4.2) has in general a high condition number. However, the observed accuracy should be very appropriate for many applications. 


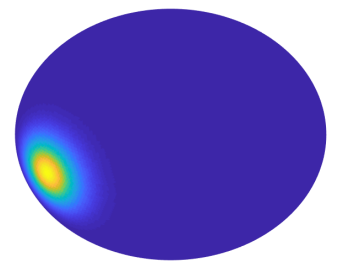

(a)

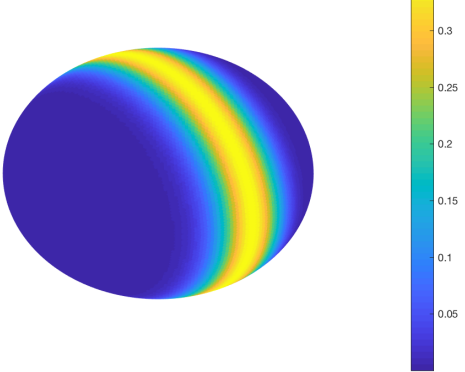

(b)

Fig. 4.1. Exact steady state solution for (a) uniaxial extensional motion and (b) biaxial extensional motion using $D_{r}=0.1$.
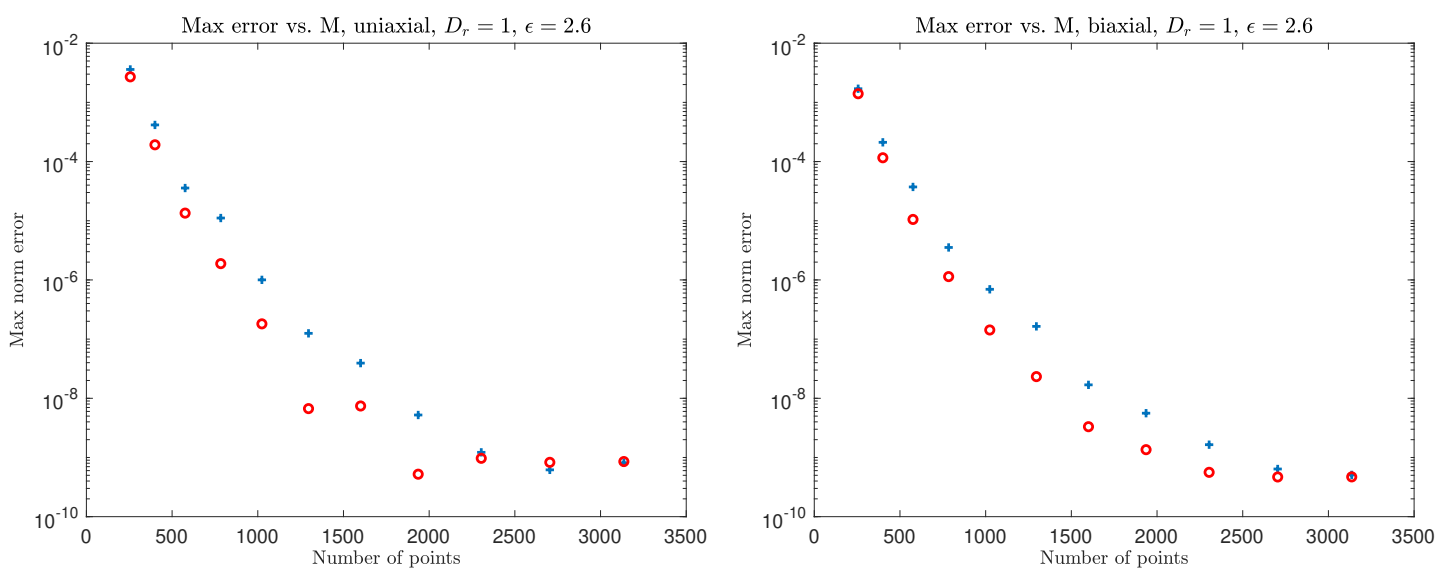

Fig. 4.2. Max-norm error vs. $M$ for steady state computations of (left) uniaxial and (right) biaxial extensional motion using $D_{r}=1$ and $\epsilon=2.6$. The blue crosses indicate the error obtained by using the MD nodes, the red circles indicate the error using the ME nodes.

To further investigate the accuracy of the RBF method, Table 4.1 shows more data of an accuracy study for Example 4.1 using $\ell=1, D_{r}=1$ and ME nodes. Now we consider different values of $\epsilon$ and report the max norm error as well as the condition number of the matrix (computed with the cond routine from MATLAB), which describes the ode in Equation (4.6), for different numbers of nodes $N$. Smaller values of $\epsilon$ increase the accuracy but lead to a larger condition number. This larger condition number causes instabilities. While larger values of $\epsilon$ increase the stability, the accuracy obtained at nodes is in all considered cases limited by about $8.5 \cdot 10^{-10}$. This accuracy is however considerably higher than the accuracy obtained with the second order finite volume method on grids with a comparable resolution, see [8].

In our second test problem we consider shear flow.

Example 4.2. We consider the initial value problem

$$
\begin{aligned}
& \partial_{t} f(t, n)+\nabla_{n} \cdot\left(P_{n^{\perp}} \nabla_{x} u_{e x t} n f(t, n)\right)=D_{r} \Delta_{n} f(t, n), \\
& f(0, n)=1 / 4 \pi
\end{aligned}
$$



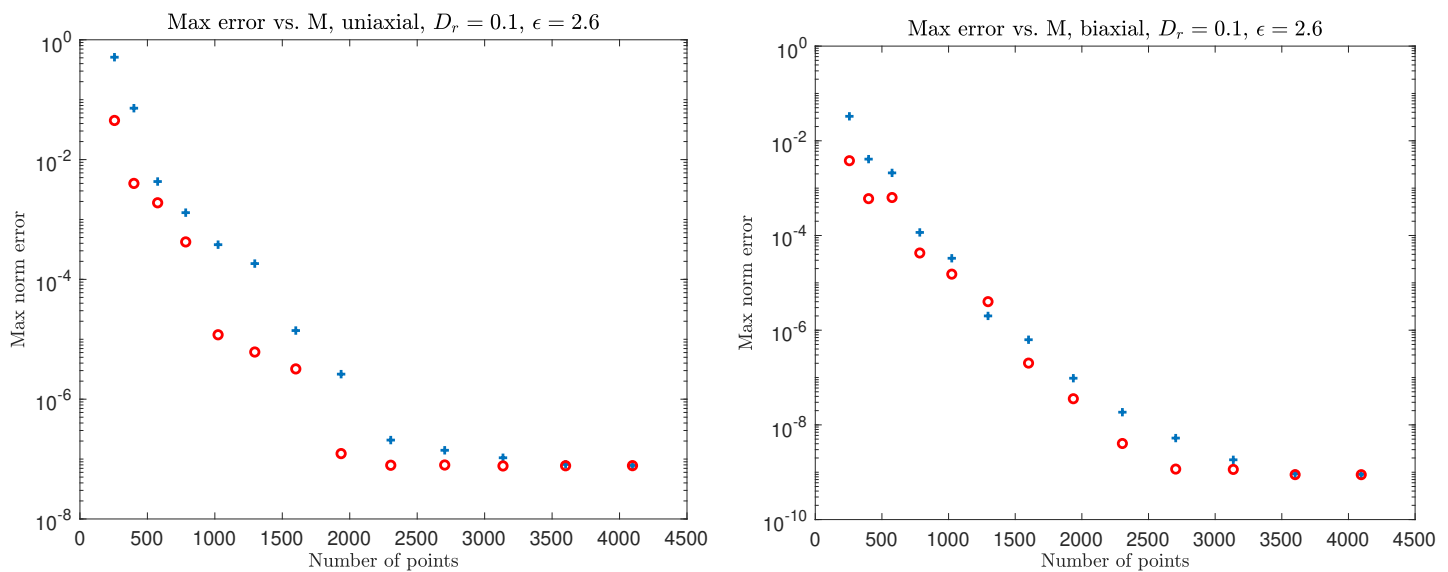

Fig. 4.3. Max-norm error vs. $M$ for steady state computations of (left) uniaxial and (right) biaxial extensional motion using $D_{r}=0.1$ and $\epsilon=2.6$. The blue crosses indicate the error obtained by using the MD nodes, the red circles indicate the error using the ME nodes.

Table 4.1: Accuracy study for the test problem specified in Example 4.1 with $D_{r}=1, \ell=1, T=10$ and MD nodes. We show the $\|\cdot\|_{\infty}$ norm error and the condition number for different values of $\epsilon$.

\begin{tabular}{|c|c|c|c|c|c|c|}
\hline \multirow{2}{*}{$N$} & \multicolumn{3}{|c|}{ error in $\|\cdot\|_{\infty}$ norm } & \multicolumn{3}{c|}{ condition number } \\
\cline { 2 - 7 } & $\epsilon=1$ & $\epsilon=2$ & $\epsilon=3$ & $\epsilon=1$ & $\epsilon=2$ & $\epsilon=3$ \\
\hline 256 & $2.731 \cdot 10^{-8}$ & $1.277 \cdot 10^{-4}$ & $1.079 \cdot 10^{-2}$ & $1.227 \cdot 10^{11}$ & $6.757 \cdot 10^{6}$ & $7.067 \cdot 10^{4}$ \\
400 & $9.855 \cdot 10^{-10}$ & $3.620 \cdot 10^{-6}$ & $1.153 \cdot 10^{-3}$ & $6.267 \cdot 10^{12}$ & $3.836 \cdot 10^{8}$ & $1.043 \cdot 10^{6}$ \\
784 & $8.446 \cdot 10^{-10}$ & $3.794 \cdot 10^{-8}$ & $1.609 \cdot 10^{-5}$ & $1.760 \cdot 10^{16}$ & $6.776 \cdot 10^{10}$ & $1.444 \cdot 10^{8}$ \\
1296 & $8.461 \cdot 10^{-10}$ & $1.042 \cdot 10^{-9}$ & $1.773 \cdot 10^{-7}$ & $2.814 \cdot 10^{17}$ & $1.408 \cdot 10^{13}$ & $2.537 \cdot 10^{10}$ \\
1600 & unstable & $7.717 \cdot 10^{-10}$ & $7.631 \cdot 10^{-8}$ & $3.475 \cdot 10^{18}$ & $6.052 \cdot 10^{13}$ & $6.280 \cdot 10^{10}$ \\
4096 & & $8.456 \cdot 10^{-10}$ & $8.553 \cdot 10^{-10}$ & & $2.490 \cdot 10^{17}$ & $9.852 \cdot 10^{14}$ \\
4624 & & unstable & $8.4157 \cdot 10^{-10}$ & & $8.800 \cdot 10^{16}$ & $2.728 \cdot 10^{15}$ \\
\hline
\end{tabular}

with $n \in S^{2}$ and an externally imposed velocity gradient which corresponds to shear flow, i.e.

$$
\nabla_{x} u_{e x t}:=\left(\begin{array}{ccc}
0 & u_{y} & 0 \\
0 & 0 & 0 \\
0 & 0 & 0
\end{array}\right) .
$$

In Fig. 4.4, we show numerical results of Example 4.2 obtained with the RBF method for different values of $u_{y}$ and different values of $M$. The numerical results obtained for $M=400$ agree already very well with the more resolved computations that used $M=1600$. The black dots in these figures show the ME points, which were used in these simulations.

Recall from the full model (2.1)-(2.3), that the microscopic solution $f$ influences the macroscopic flow $u$ via the stress tensor $\sigma$. In order to obtain efficient approximations of the full model it is desirable to compute accurate approximations of $f$ using relatively few degrees of freedom, i.e. $M$ should be relatively small. For shear flow of the form $u=(u(y), 0,0)^{T}$, the component $\sigma_{12}=\sigma_{21}$ of the stress tensor (2.2) is relevant. This component has the form

$$
\sigma_{12}=3 \int_{S^{2}} x y f_{e q}(n) d n
$$

where $x$ and $y$ are the first and second components of $n \in S^{2}$. 

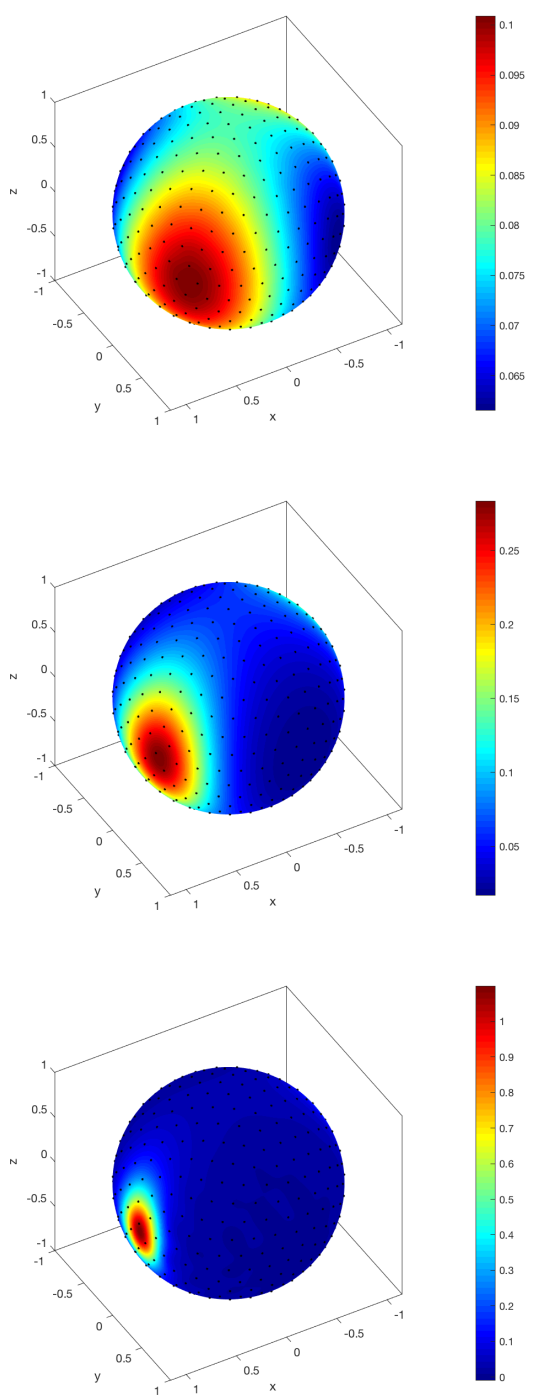
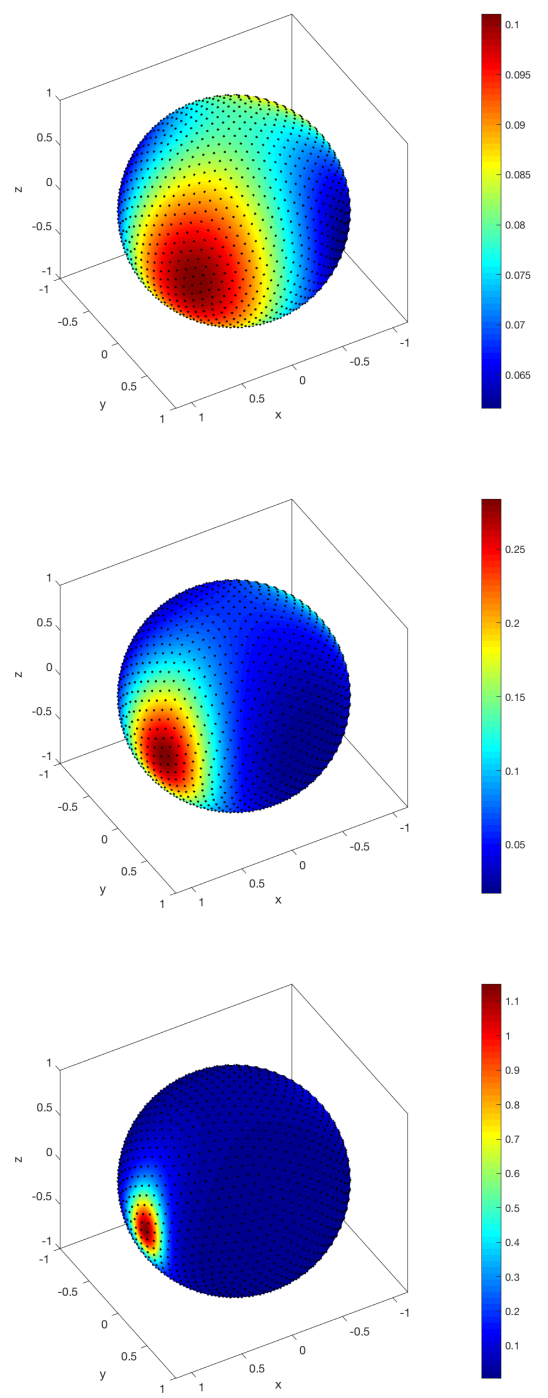

Fig. 4.4. Shear flow solution for (top) $u_{y}=1$, (middle) $u_{y}=10$ and (bottom) $u_{y}=100$. For all of these computations we used $\epsilon=2.6$ and $D_{r}=1$. For the plots on the left hand side we used $400 \mathrm{ME}$ nodes and for the plots on the right hand side we used $1600 \mathrm{ME}$ nodes. Note that the $x$-axis describes the flow direction, the $y$-axis describes the shear direction and the $z$-axis describes the vorticity direction.

For a constant externally imposed velocity gradient $\nabla_{x} u_{e x t}$ and a constant rotational diffusion coefficient $D_{r}$, the Smoluchowski equation reaches a steady state. For constant $D_{r}$ and constant initial values (here we used $f(0, n)=1 /(4 \pi)$ ), this defines a mapping from $\nabla_{x} u_{e x t}$ to $\sigma$, where $\sigma$ is computed using the steady state solution of $f$. For shear flow, the mapping from $u_{y}$ to $\sigma_{12}$ is relevant. It is known, see [12], that this mapping is non-monotone, which leads to a complex solution structure of the coupled problem (even for shear flow).

Starting with $f(n, 0)=1 /(4 \pi)$, we compute solutions of the Smoluchowski equation at time $T=10$ for $D_{r}=1$ and different values of $u_{y}$, with $-100 \leq u_{y} \leq 100$. At time $T=10$ the numerical solution has reached a steady state, which was used in (4.8) to compute the 
stress component $\sigma_{12}$. In Fig. 4.5, we show results of this mapping from shear velocity to the stress component. The Smoluchowski equation was solved using the RBF method with different numbers of $\mathrm{ME}$ node points, i.e. we used $M=225, M=400$ and $M=900$. The solid black line in Fig. 4.5 is a reference solution, which we obtained by using $M=2500$. For $M=400$, which is still a relatively coarse approximation, we already obtain very good results. The very coarse approximation, using only $M=225$ nodes on the sphere, was not sufficient. Note that weights of a numerical quadrature formula are provided together with the RBF nodes, see [15].
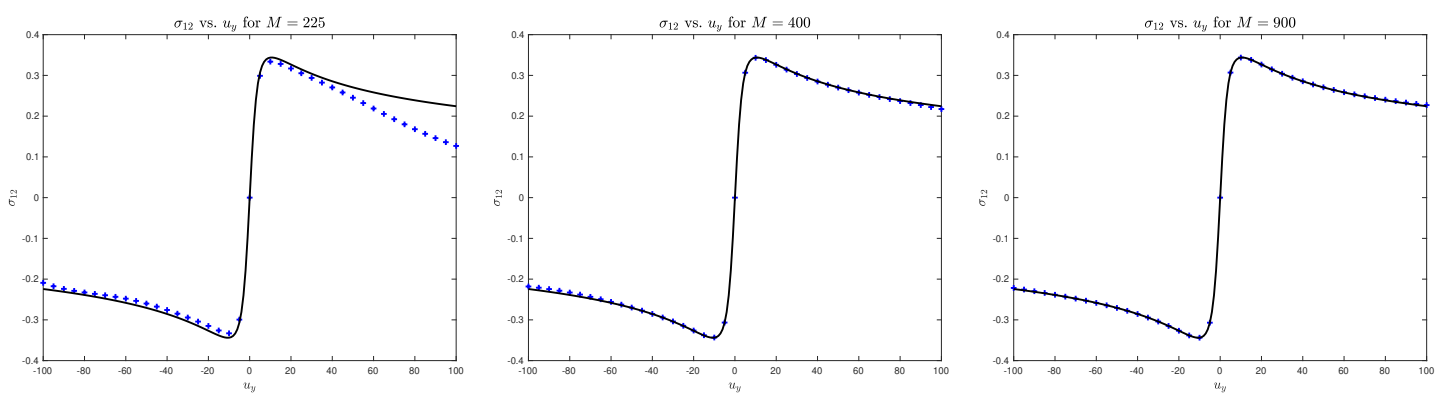

Fig. 4.5. The stress component $\sigma_{12}$ as a function of $u_{y}$ for steady state shear flow computations of the Smoluchowski equation. We used $M=225$ (left), $M=400$ (middle) and $M=900$ (right) for the solution of the Smoluchowski equation. The black line is a reference solution obtained by using $M=2500$.

We conclude that RBF methods are attractive numerical methods for these kind of applications. They reach an accuracy, using relatively few degrees of freedom, which is comparable with the accuracy of spectral methods. Furthermore, the derivation of these methods for new applications is simpler.

Acknowledgments. We thank Natasha Flyer from the National Center for Atmospheric Research Boulder, USA, for helpful discussions and for her Matlab code to solve the advection diffusion equation on the sphere using radial basis functions.

\section{References}

[1] C. Bechinger, R. Di Leonardo, H. Löwen, C. Reichhardt, G. Volpe and G. Volpe, Active particles in complex and crowded environments Reviews of Modern Physics, 88 (2016).

[2] M.D. Buhmann, Radial basis functions. Acta Numerica, (2000), 1-38.

[3] M. Doi and S.F. Edwards, The Theory of Polymer Dynamics. Oxford University Press, 1986.

[4] N. Flyer and G.B. Wright, Transport schemes on a sphere using radial basis bunctions, J. Comput. Phys., 226 (2007), 1059-1084.

[5] N. Flyer and G.B. Wright, A radial basis function method for the shallow water equations on the sphere, Proc. R. Soc. A, 465 (2009), 1949-1976.

[6] B. Fornberg and N. Flyer, A Primer on Radial Basis Functions with Applications to the Geosciences, volume 87 of CBMS-NSF Regional Conference Series in Applied Mathematics, Society for Industrial and Applied Mathematics (SIAM), Philadelphia, PA, 2015.

[7] B. Fornberg and C. Piret, On choosing a radial basis function and a shape parameter when solving convective pde on a sphere, J. Comput. Phys., 227 (2008), 2758-2780.

[8] C. Helzel and F. Otto, Multiscale simulations for suspensions of rod-like molecules, J. Comput. Phys., 216 (2006), 52-75. 
[9] C. Helzel and A.E. Tzavaras, A kinetic model for the sedimentation of rod-like particles, Multiscale Model Simul., 15 (2017), 500-536.

[10] D.J. Knezevic and E. Süli, A heterogeneus alternating-direction method for a micro-macro dilute polymeric fluid model, ESAIM: M2AN, 43 (2009), 1117-1156.

[11] C.A. Micchelli, Interpolation of scattered data: Distance matrices and conditionally positive definite functions, Const. Approx., 2 (1986), 11-22.

[12] F. Otto and A.E. Tzavaras, Continuity of velocity gradients in suspensions of rod-like particles, Commun. Math. Phys., 277 (2008), 729-758.

[13] C. Piret, The orthogonal gradients method: A radial basis functions method for solving partial differential equations on arbitrary surfaces, J. Comput. Phys., 231 (2012), 4662-4675.

[14] V. Shankar, G.B. Wright, R.M. Kirby and A.L. Fogelson, A radial basis function (rbf)-finite difference (fd) method for diffusion and reaction-diffusion equations on surfaces. J. Sci. Comput., 63 (2015), 745-768.

[15] R. Womersley, Interpolation and cubature on the sphere, http://web.maths.unsw.edu.au/rsw/ Sphere/.

[16] G.B. Wright, N. Flyer and D.A. Yuen, A hybrid radial basis function-pseudospectral method for thermal convection in a 3-d spherical shell, Geochemistry, Geophysics, Geosystems, 11 (2010), Q07003. 